\title{
Can Chatbots Help Support a Person's Mental Health? Perceptions and Views from Mental Healthcare Professionals and Experts
}

\author{
COLM SWEENEY, COURTNEY POTTS, EDEL ENNIS, RAYMOND BOND, \\ MAURICE D. MULVENNA, and SIOBHAN O'NEILL, Ulster University \\ MARTIN MALCOLM, NHS Western Isles \\ LAURI KUOSMANEN, University of Eastern Finland \\ CATRINE KOSTENIUS, Luleå University of Technology \\ ALEX VAKALOUDIS, Cork Institute of Technology \\ GAVIN MCCONVEY, Action Mental Health, \\ ROBIN TURKINGTON and DAVID HANNA, Ulster University \\ HEIDI NIEMINEN and ANNA-KAISA VARTIAINEN, University of Eastern Finland \\ ALISON ROBERTSON, NHS Western Isles \\ MICHAEL F. MCTEAR, Ulster University
}

\begin{abstract}
The objective of this study was to understand the attitudes of professionals who work in mental health regarding the use of conversational user interfaces, or chatbots, to support people's mental health and wellbeing. This study involves an online survey to measure the awareness and attitudes of mental healthcare professionals and experts. The findings from this survey show that more than half of the participants in the survey agreed that there are benefits associated with mental healthcare chatbots $(65 \%, \mathrm{p}<0.01)$. The perceived importance of chatbots was also relatively high $(74 \%, \mathrm{p}<0.01)$, with more than threequarters $(79 \%, \mathrm{p}<0.01)$ of respondents agreeing that mental healthcare chatbots could help their clients better manage their own health, yet chatbots are overwhelmingly perceived as not adequately understanding or displaying human emotion (86\%, $\mathrm{p}<0.01)$. Even though the level of personal experience with chatbots among professionals and experts in mental health has been quite low, this study shows that where they have been used, the experience has been mostly satisfactory. This study has found that as years of experience increased, there was a corresponding increase in the belief that healthcare chatbots could help clients better manage their own mental health.
\end{abstract}

The ChatPal consortium acknowledges the support provided by the Interreg VB Northern Periphery \& Arctic Programme under the grant for Conversational Interfaces Supporting Mental Health and Wellbeing of People in Sparsely Populated Areas (ChatPal) project number 345. Authors' addresses: C. Sweeney, E. Ennis, and S. O’Neill, School of Psychology, Ulster University, Northland Road, Derry-Londonderry, UK; emails: \{sweeneyc23, e.ennis, sm.oneill\}@ulster.ac.uk; C. Potts, R. Bond, M. D. Mulvenna, and R. Turkington, School of Computing, Ulster University, Shore Road, Newtownabbey, UK; emails: \{c.potts, rb.bond, md.mulvenna, turkington-r\}@ulster.ac.uk; M. Malcolm and A. Robertson, Dept. Public Health Intelligence, NHS Western Isles, Stornoway, Isle of Lewis, UK; emails: \{martin.malcolm2, alison.robertson13\}@nhs.scot; L. Kuosmanen and H. Nieminen, Department of Nursing Science, University of Eastern Finland, Kuopio Campus / Canthia, Yliopistonranta 1C, 70211 Kuopio, Finland; emails: \{lauri.kuosmanen, heidi.nieminen\}@uef.fi; C. Kostenius, Department of Health, Education and Technology, Luleå University of Technology, Luleå, Sweden; email: catrine.kostenius@ltu.se; A. Vakaloudis, Nimbus Research Centre, Cork Institute of Technology, Cork, Ireland; email: alex.vakaloudis@cit.ie; G. McConvey, Action Mental Health, Jubilee Road, Newtownards, UK; email: gmcconvey@amh.org.uk; D. Hanna, Department of Global Business Enterprise, Ulster University, Cromore Road, Coleraine, UK; email: da.hanna@ulster.ac.uk; A.-K. Vartiainen, Department of Nursing Science and Department of Health and Social Management, University of Eastern Finland Kuopio Campus / Canthia, Yliopistonranta 1C, 70211 Kuopio, Finland; email: anna-kaisa.vartiainen@uef.fi; M. F. McTear, School of Computing, Ulster University, Shore Road, Newtownabbey, UK; email: mf.mctear@ulster.ac.uk.

Permission to make digital or hard copies of all or part of this work for personal or classroom use is granted without fee provided that copies are not made or distributed for profit or commercial advantage and that copies bear this notice and the full citation on the first page. Copyrights for components of this work owned by others than ACM must be honored. Abstracting with credit is permitted. To copy otherwise, or republish, to post on servers or to redistribute to lists, requires prior specific permission and/or a fee. Request permissions from permissions@acm.org. (C) 2021 Association for Computing Machinery.

$2637-8051 / 2021 / 07-A R T 25 \$ 15.00$

https://doi.org/10.1145/3453175 
CCS Concepts: • Human-centered computing $\rightarrow$ Human computer interaction $(H C I)$; Applied computing $\rightarrow$ Health informatics; Health care information systems; Consumer health;

Additional Key Words and Phrases: Mental health professionals, chatbots, conversational user interfaces, mental health, mental health survey, wellbeing

\section{ACM Reference format:}

Colm Sweeney, Courtney Potts, Edel Ennis, Raymond Bond, Maurice D. Mulvenna, Siobhan O'neill, Martin Malcolm, Lauri Kuosmanen, Catrine Kostenius, Alex Vakaloudis, Gavin Mcconvey, Robin Turkington, David Hanna, Heidi Nieminen, Annakaisa Vartiainen, Alison Robertson, and Michael F. Mctear. 2021. Can Chatbots Help Support a Person's Mental Health? Perceptions and Views from Mental Healthcare Professionals and Experts. ACM Trans. Comput. Healthcare 2, 3, Article 25 (July 2021), 15 pages.

https://doi.org/10.1145/3453175

\section{INTRODUCTION}

Chatbots, also known as conversational user interfaces, are tools that use machine learning and artificial intelligence to simulate human communication, either through voice or text communication.

Voice-based chatbots are called upon within mobile devices, computers, and smart speakers such as Amazon Alexa and Google Home. Text-based chatbots can be accessed through many channels, such as Messenger, Kik, Slack, and Telegram, or in a web or mobile application [5]. The user can converse with the chatbot using text or "quick replies" (buttons). Voice-based chatbots, used at home for support of mental health and wellbeing, require translation from speech to text (and vice versa) with a success rate dependent on accent and surrounding sound (e.g., machine operating, TV on, other people talking). Additionally, elements of conversation such as secure authentication, selection over a large number of options, and transcription of special terms such as names and emails can more easily be managed with text-based rather than voice-based conversation.

They support interactive communications between persons and a computer program [9]. The earliest chatbot dating back to the 1960s was ELIZA, and it was developed to try to mimic the responses of a psychotherapist in a therapy session [30]. It made use of pattern matching and pronoun substitution to give an illusion of understanding even though it had no built-in knowledge [16]. Computing technology has advanced since then, with the advances in artificial intelligence and machine learning, and in particular deep neural networks, which have led to further development of chatbots. In addition, the increased adoption of mobile Internet and messaging platforms has driven the adoption of digital interventions such as chatbots [11].

Chatbots operate using natural language, and they have gained a lot of traction recently because they have the potential to provide a multitude of services through one point of contact. Chatbots have been used in education [32], customer service innovations [27], and healthcare [8]. Within the area of mental health, there has been a recent drive toward creating digital interventions that can either supplement or replace face-to-face mental health services [15]. As access to mental health services remains an issue worldwide, with the mental health workforce insufficient to meet the growing demand for mental health services [29], automated procedures hold great promise for both mental healthcare providers and for those in need of mental health services [13].

Many chatbots are available for use in mental health care provision. One of the better-known mental health chatbots is Woebot [10], which is a chatbot therapist. It works using cognitive behavioral therapy and applies techniques to help clients improve their mental health. The results of a randomized controlled trial among students found that Woebot significantly reduced symptoms of depression within 2 weeks [10].

This conversational agent was launched via Facebook Messenger, but there were some criticisms with this, as Facebook, and not Woebot or the individual, owned the conversation data [23]. This is more relevant recently, where Facebook has been under fire for the company's handling of personal data [28]. There were also criticisms that the Woebot app failed to respond appropriately to reports of child sexual abuse resulting in the Children's 
Commissioner for England deeming the app not fit for purpose [18]. To address some of these issues, and particularly in relation to the privacy issues with this application, users can now ask the Woebot app to delete their user history, as well as remove all conversations, once the conversation has ended [28].

iHelpr is another mental health chatbot developed to provide self-assessment and guidelines for stress, anxiety, depression, sleep, and self-esteem [5]. A study was conducted regarding its use, via questionnaire, and it was found that the respondents found the chatbot to be enjoyable and easy to use and stated that there is a consistent personality throughout the conversation, which they liked [5]. Tess is another chatbot designed to provide support for treating anxiety and depression, and it was shown that it is a feasible option for offering support for depression [12]. Bibliotherapy is another method of mental health support. This is a form of talking therapy that uses short stories to support people with mental health issues, using concepts of cognitive behavioral therapy to assist in the recovery of the client, and recent research has been investigating its adoption by bibliotherapy facilitators [19].

Various studies have been carried out to determine the feasibility and efficacy of chatbot use. An early study in 2017 [4] used an online questionnaire to determine why chatbot users in the United States were using chatbots. The results showed that the greatest motivational factor was productivity, in that chatbots helped users obtain timely assistance or information [4]. A recent survey conducted by Palanica et al. [24] set out to determine the opinions of medical practitioners on the use of this technology in healthcare. Their study showed that chatbots may have a beneficial role to play in healthcare support [24].

Current literature demonstrates that there are widespread benefits to using chatbots. One is that it is preferable for some people to use chatbots to complete certain tasks normally performed on a web page or mobile application [11]. Chatbots are also seen to relieve certain barriers in mental health provision, such as stigma around accessing psychological health services and geographical isolation that can hinder attendance at faceto-face counseling [20]. People working unconventional shifts may also have problems gaining access to mental health services. Chatbots could be used as a potential solution to these problems, as mobile mental health interventions are nonintrusive and easily accessible for anyone with a mobile phone. There has also been some evidence to suggest that chatbots can be effective in dealing with individuals with major depressive disorder [29]. Mental health counseling can be improved through the use of chatbots, as chatbots can provide a user with instant information [6], they have 24/7 availability, and they will provide cost savings for the users in the form of travel expenses and telephone charges [14]. In addition, users enjoy the anonymity offered by chatbots, as some patients were shown to disclose sensitive information to a chatbot but they would not have done so with a human therapist, as demonstrated by Lucas et al. [17]. In the United Kingdom, Samaritans, the helpline service, recorded that users younger than 25 years were the highest user age group of their text messaging service, and they were more likely to communicate through text than to phone the organization [26]. These young people can also feel uncomfortable disclosing their feelings to a human being [29]. As a recent study shows, in some circumstances, patients are more likely to disclose personal details to artificial intelligence-powered conversational assistants rather than to an actual clinician [21]. Many young people also feel that their problems are too personal, or they fear that this sensitive data could be shared with others [31].

Chatbots also have their limitations. They may not be able to understand the nuances of human language. Artificial intelligence has to understand the complexities at play, to prevent the provision of an unsuitable answer or by providing unclear or nonsensical responses, to be able to communicate what is at the core of mental health problem. Chatbots could offer reminders every now and again that they are in fact an "artificial intelligence" to counter any confusion. As the Woebot application warns its users: "As smart as I may seem, I'm not capable of really understanding what you need" [15]. Many are dismayed and horrified at the concept of chatbots for mental health and in particular for the area of suicide prevention, arguing that such a complex problem cannot conceivably be reduced to a computer algorithm [2]. Chatbots also fall short in the provision of mental health support for sensitive topics, such as for suicide risks and where abuse is being reported [28]. Indeed, there is not enough evidence-based data to support chatbot use in suicide prevention [25]. 
Privacy is also a major concern for users of these applications, and developers need to ensure that data sharing will not expose users to privacy risks. Poor adherence seems to be another problem with digital mental health interventions [7], and for those people who do go on to maintain a relationship with these digital resources, the fear is that some vulnerable people may begin to rely on them too much, which may lead to anxiety when these applications are not available. Overall, there is a need for a wider discussion about how all mental health services can and should encourage the safe and ethical use of chatbots [29].

In the recent study by Palanica et al. [24], challenges were perceived due to chatbots not being able to understand or display human emotion. The majority of physicians (70\%) in this survey expressed their concern about risks associated with healthcare chatbots for patients[24], and these concerns relate to chatbots not being able to understand the complex use of language associated with a mental health crisis in such a way to recognize symptoms and respond appropriately [18]. Hence, rather than developing something that is state of the art, the developers may need to ensure that the technology is centered on understanding the needs of someone in crisis [3].

Chatbots represent a shift in how people manage their mental health. Although there has been an increase in the development and use of such applications, it is not fully understood why professionals and experts in mental health would use chatbots and encourage their use by their clients. The study by Palanica et al. [24] investigated how the use of healthcare chatbots are regarded by physicians. Their survey concluded that chatbots can have a beneficial role to play in healthcare support but that chatbots currently do not have the expert medical knowledge needed to replace the role of the traditional physician. The effectiveness of chatbot use in the area of mental health has not been fully researched [22], and this survey extends the work of Palanica et al. [24] to look at the professionals and experts who work in the area of mental health, to determine if chatbots designed for mental health are beneficial and to determine the efficacy of such tools.

Mental healthcare professionals are crucial in advising clients on how best to protect their wellbeing, but little is known about how they perceive chatbots in terms of benefits, challenges, and risks to patients [24]. In the survey by Palanica et al. [24], clinicians from the United States suggest that chatbots may be important in the self-management of health (e.g., for appointment scheduling or medication monitoring). But these clinicians were concerned that chatbots do not understand human emotions and cannot provide diagnosis, as they do not have expert knowledge and do not know the overall person and also may encourage self-diagnosis within clients [24]. It was felt that chatbots may play a role in supporting, motivating, and coaching patients, but even in this they are limited by the fact that they cannot display or understand human emotion [24]. The survey showed that clinicians thought chatbots could not replace healthcare professionals, but they could support them, and more education on evidence-based research surrounding chatbots is necessary [24]. Andersson [1] made similar findings in which this type of technology was found to have poor adoption rates by physicians and poor adherence by patients.

\section{OBJECTIVES}

The research of Palanica et al. [24] involved physicians in the United States only [24], where the opinions were primarily about physical health. Further research is needed to consider different mental healthcare professionals in different environments and how they perceive chatbots for use with their clients. Therefore, the main objective of this survey is to assess awareness, attitudes, and practices of mental healthcare professionals regarding the use of digital health tools and particularly chatbots to augment and improve mental health service provision. The survey investigates the use of healthcare chatbots, perceived benefits of healthcare chatbots to clients, perceived challenges of healthcare chatbot usage, perceived risks of healthcare chatbots, and the perceptions of healthcare chatbots in the role of a mental healthcare professional. An additional objective is to determine if there is an association between a mental healthcare professional's experience and the professional's likelihood to recommend or prescribe the use of mental healthcare chatbots to clients. 


\section{METHODS}

The study involved an online survey of awareness, attitudes, and practices relating to digital technology among mental healthcare professionals. Ethical approval for this study was obtained from the School of Psychology Filter (Ethics) Committee and the University Ethics Review Board at Ulster University. Respondents gave informed consent at the entry point to the online survey.

The survey was part of a larger project known as ChatPal, a project funded by the Northern Periphery and Arctic Programme (2014-2020) to co-create a chatbot to support the mental wellbeing of people living in sparsely populated areas.

The ChatPal consortium is made up of partners from Northern Ireland, Ireland, Scotland, Sweden, and Finland. Initial recruitment for the survey took place in Northern Ireland, via local mental health services organization and ChatPal partner Action Mental Health, which shared the survey with their staff via email, emphasizing the voluntary nature of participating. Recruitment also took place via email by academic institutions and healthcare services across the consortium's partnership networks. Snowball sampling was used to recruit additional mental health professionals across each of these regions in Europe.

Only mental healthcare professionals were eligible to participate in the survey. This included individuals employed to either support the mental well-being of the population in general or support those with mental ill health on a practical or an academic basis.

The survey is subdivided into five main sections: (1) usage of mental healthcare chatbots, (2) perceived benefits of healthcare chatbots to clients, (3) perceived challenges of mental healthcare chatbot usage, (4) perceived risks of healthcare chatbots, and (5) perceptions of mental healthcare chatbots by mental healthcare professionals. The survey categories remain similar to the survey designed by Palanica et al. [24], but the emphasis on physical health and the associated symptomatology has been changed to mental health for this current study. In addition, references to professionals and technology have been made more relevant to mental health rather than physical health.

Data were analyzed using descriptive analyses to examine the characteristics of participant responses to survey items on mental healthcare chatbots. Data analysis was carried out using the Python programming language and Python Jupyter Notebook. Spearman's rank correlation coefficients were used for association analysis between variables. Significance testing was computed for correlations, where $p<.05$ was considered statistically significant. Bar charts displaying the survey results contain error bars with an error amount of $5 \%$ for each data point, as a percentage of the value of that data point.

\section{RESULTS}

\subsection{Use of Mental Health Chatbots}

When asked about the perceived importance of chatbots (Figure 1), 77\% of professionals and experts in mental health thought that chatbots are either somewhat important (66\%) or very important (11\%). In contrast, $23 \%$ believed that chatbots are somewhat unimportant $(20 \%)$ or very unimportant $(3 \%)$.

The surveyed professionals and experts in mental healthcare were also provided with a list of some of the chatbots currently available for use in mental healthcare and asked about their levels of awareness of these chatbots. From their replies, it is noticeable that most of the respondents have neither heard of nor used any of these applications. Of the 16 respondents who had used these chatbots, $24 \%$ of the respondents believed that their clients use mental healthcare chatbots (with 35\% not using them) with the majority (38\%) being unsure or did not know if their clients used mental healthcare chatbots.

The respondents were also asked if they would be likely to prescribe the use of mental health chatbots to their clients within the next 5 years. A total of $80 \%$ of respondents replied that they would be very likely (24\%) or somewhat likely (56\%) to prescribe these tools (Figure 2). Although there is a disparity in gender distribution in 


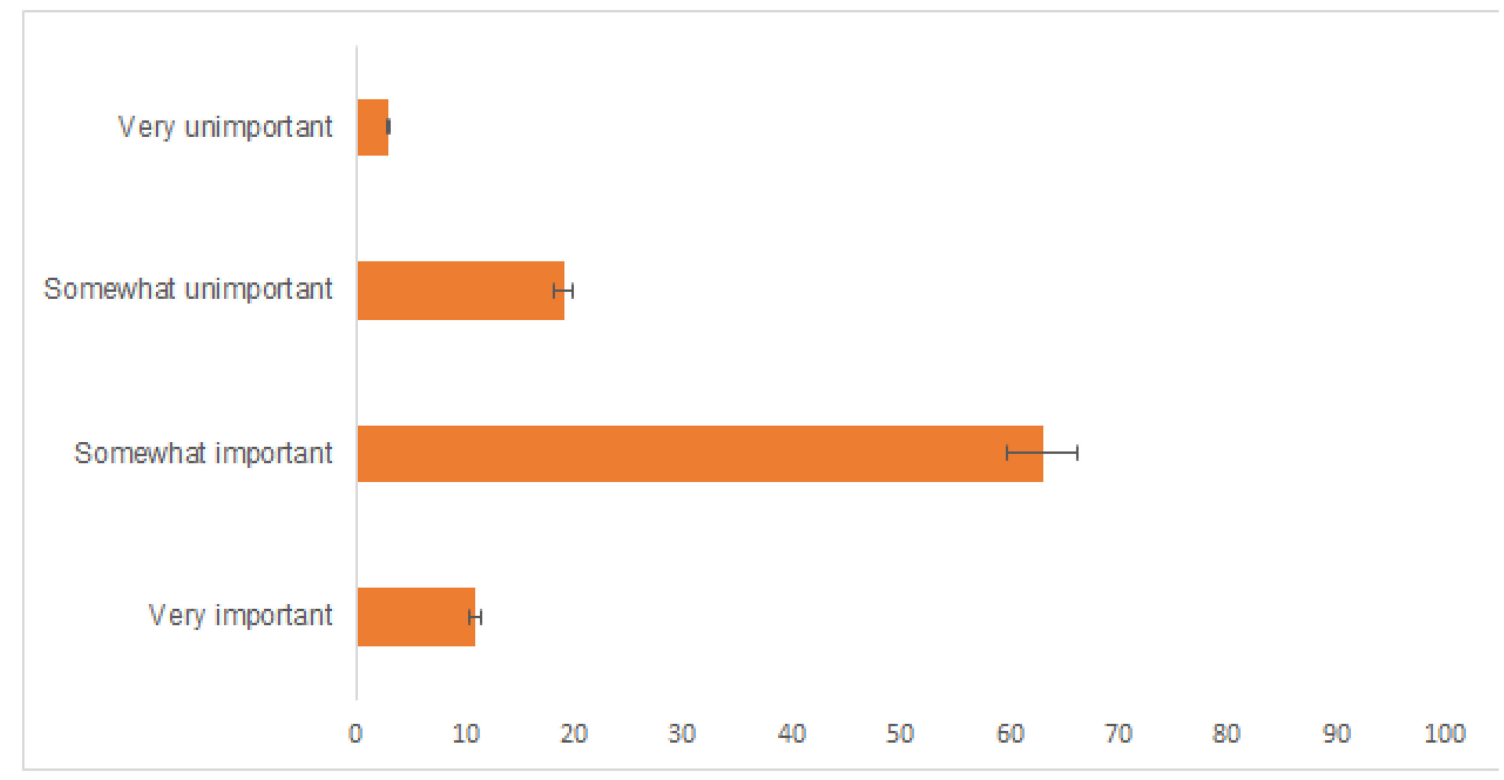

Fig. 1. Perceived importance of chatbots in mental healthcare.

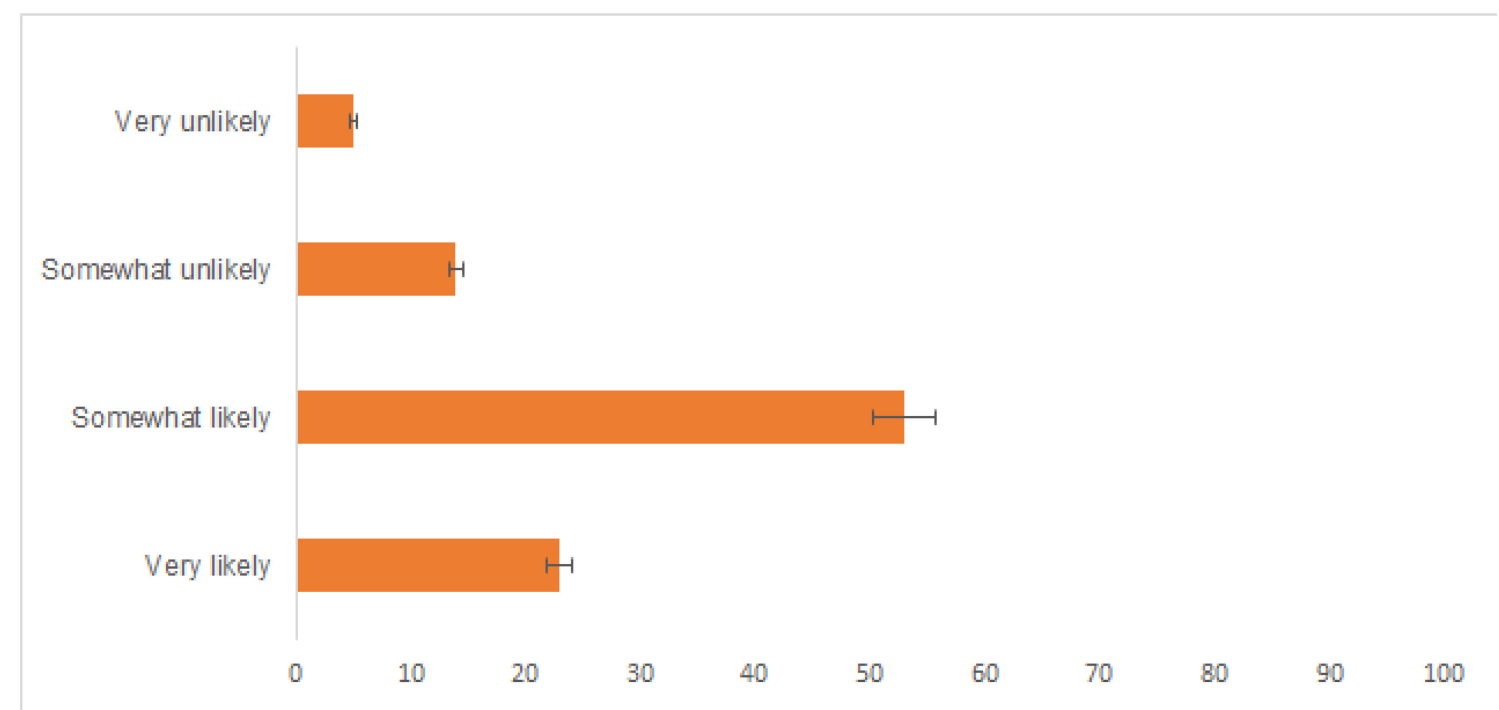

Fig. 2. Perceived likelihood to prescribe the use of mental healthcare chatbots to their clients within the next 5 years.

the respondents ( $16 \%$ male and $62 \%$ female), there was no significant difference between the percentage of males versus females and their likelihood to prescribe the use of mental health chatbots to their clients.

\subsection{Perceived Potential Benefits of Using Mental Healthcare Chatbots for Clients}

The perceived benefit to clients is an important indicator of the efficacy of this new technology. The professionals and experts in mental health were asked about any apparent benefits that existed with the use of chatbots in 


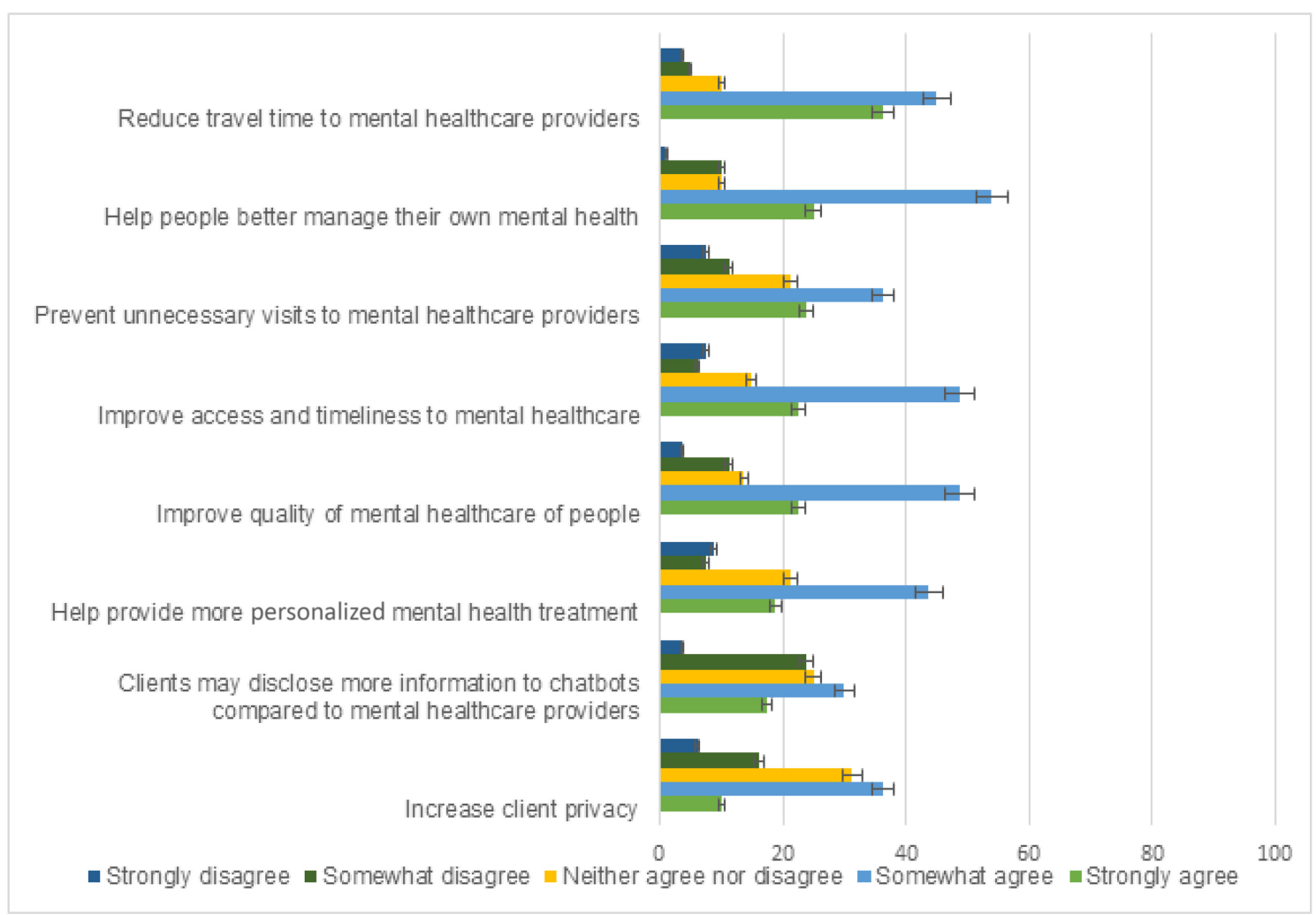

Fig. 3. Perceived benefits of mental healthcare chatbots for clients (ordered in descending order by percentage who strongly agree).

the area of mental health. Figure 3 shows an average of $65 \%(\mathrm{SD}=13.24)$ agreed to some extent that there are benefits associated with mental healthcare chatbots; nevertheless, an average of $17 \%$ (SD $=6.12$ ) disagreed to some extent that there were any potential benefits. More than three-quarters of respondents agreed that mental healthcare chatbots could help their clients better manage their own health $(79 \%)$. Chatbots were also perceived to improve access and timeliness to care $(71 \%)$ and reduce travel time to their mental healthcare providers (81\%). Sixty percent of respondents believed that mental healthcare chatbots could prevent unnecessary visits to mental healthcare providers and that clients may disclose more information to chatbots compared to mental healthcare providers $(48 \%)$.

There will also be mental health-related benefits of chatbot use for clients, with an average of $53 \%$ of respondents believing in some type of physical, psychological, or behavioral health benefit to clients, with more than half of respondents believing that mental healthcare chatbots could increase activity or exercise (59\%), improve nutrition or diet (63\%), reduce stress $(66 \%)$, or improve medication or treatment adherence $(66 \%)$.

The respondents were asked how much they believe that healthcare chatbots would help or impede their work in their daily occupational role on a scale from $0=$ "impede my work" to $5=$ "help me." The responses showed an observed range from 0 to 5 , mean of 2.95, median of 3 , and standard deviation of 1.15. The professionals and experts in mental health were also asked how likely it would be in the future for healthcare chatbots to play a more significant role in clients' health than their healthcare provider, and well over half (58\%) of respondents thought that it would most likely happen, with $27 \%$ expressing that it would be very likely. 


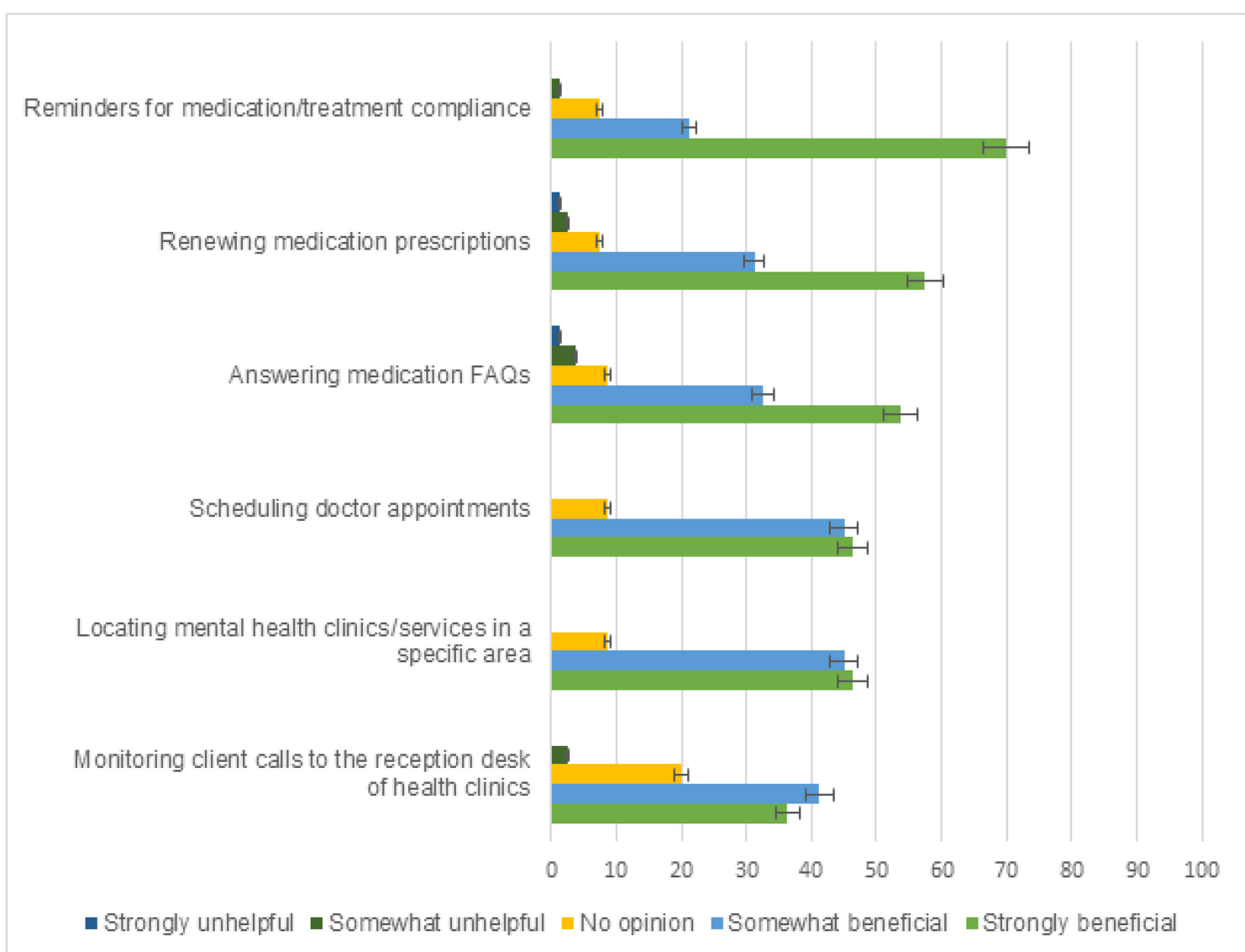

Fig. 4. Perceived logistical benefits of using chatbots for clients (ordered in descending order by percentage that find the perceptions strongly beneficial).

In relation to certain barriers to mental health provision, the majority (88\%) of professionals and experts in mental health regarded the use of chatbots as strongly beneficial or somewhat beneficial. In particular, respondents found that chatbots would be most beneficial for reminders for medication/treatment compliance (70\%), renewing medication prescriptions (58\%), and answering medication Frequently Asked Questions (54\%).

There are also perceived logistical benefits of using mental healthcare chatbots, with the majority of respondents (88\%) believing that advantages for organization, planning, and management of administrative characteristics associated with mental healthcare are either strongly or somewhat beneficial (Figure 4). Respondents perceived that chatbots would be beneficial for scheduling doctor appointments (91\%), locating mental health clinics and services in a specific area $(91 \%)$, setting reminders for medication/treatment compliance (91\%), or answering medication-related Frequently Asked Questions (86\%).

Along with the logistical benefits of using these applications for their clients, chatbots also have significant potential for use in self-management (39\%), followed by education (36\%) and training (35\%) (Figure 5).

Chatbot use, as an aid to cognitive behavioral therapy, is also perceived as having strong potential (25\%), as these convenient, intelligent tools can conceivably offer assistance at any time with little commitment from the client. The respondents also believed that chatbots could have potential to be used for particular disorders including stress $(82 \%)$, anxiety $(80 \%)$, depression $(74 \%)$, and phobia $(67.5 \%)$. And, when asked if chatbots have 


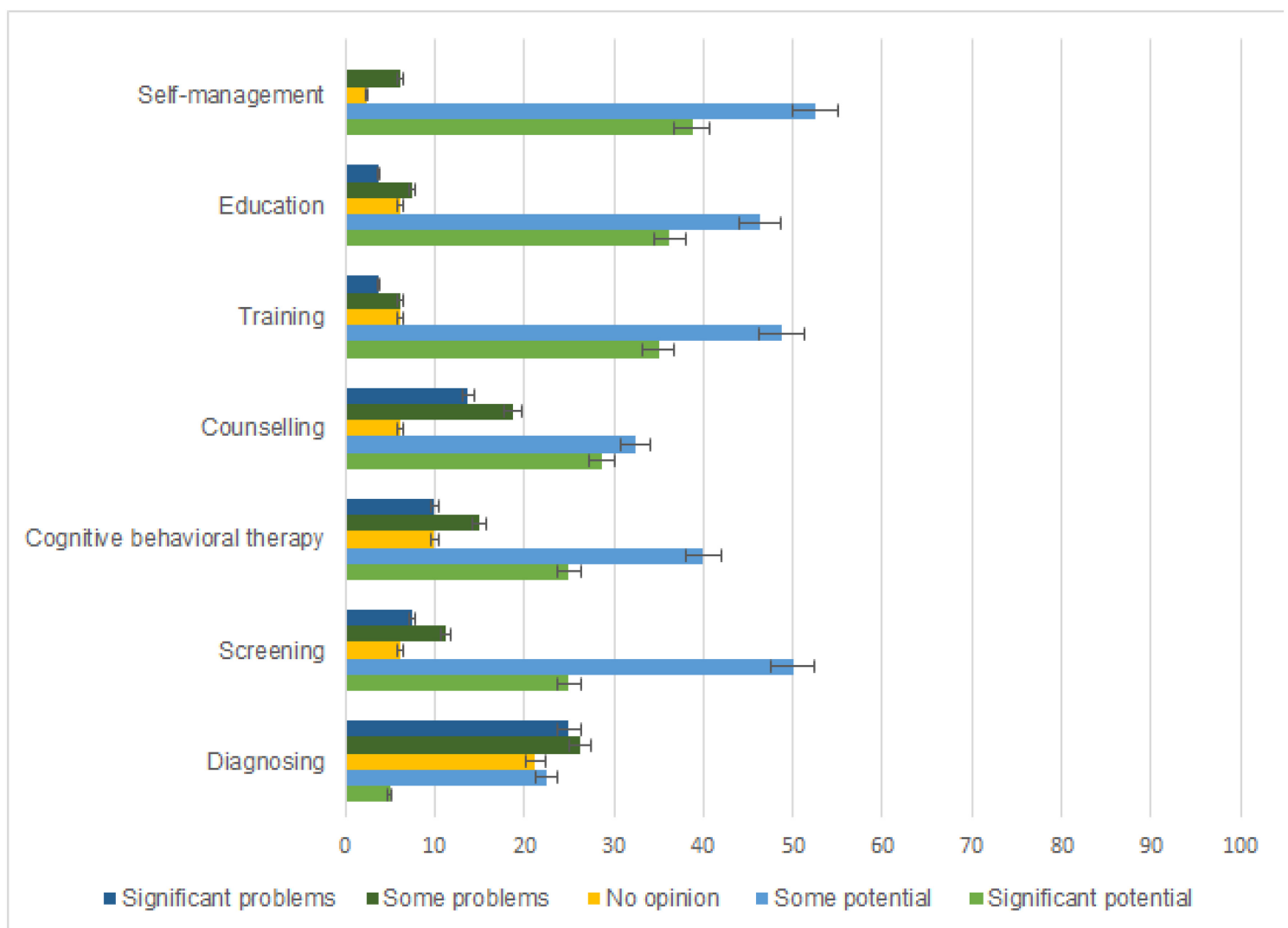

Fig. 5. Perceived potential uses for chatbots (ordered in descending order by percentage with significant potential).

the potential to improve specific health-related outcomes for clients, more than $60 \%$ of respondents replied that medication or treatment adherence, stress reduction or management, psychological well-being increase, and nutrition or diet improvement are all areas that could improve outcomes.

\subsection{Perceived Challenges and Risks Associated with Mental Healthcare Chatbots}

Along with the benefits of using mental healthcare chatbots, there are also challenges. More than half of the respondents (54\%) agree that there are various challenges associated with the use of mental healthcare chatbots for clients. The main challenge identified with the use of mental healthcare chatbots was that chatbots cannot adequately understand or display human emotion (86\%). Other challenges highlighted are that chatbots cannot effectively care to the full extent of the clients' needs (77\%), they lack the intelligence or knowledge to accurately assess clients (73\%), and respondents perceived client data privacy and confidentiality as a challenge (59\%).

This study shows overall rates of perceived risks (79\%) to be a concern to the professionals and experts in mental health, in relation to the use of chatbots in care provision (Figure 6), whereas only $6 \%$ did not agree about any perceived potential risks.

More than $75 \%$ (78\%) of respondents agreed on the listed perceived risks, with $90 \%$ claiming that the clients may not feel adequately connected to their healthcare providers as a major risk. Other major perceived risks include clients may not be able to verbalize their own mental health state clearly enough to allow the chatbot to understand $(82 \%)$, clients may not accurately understand the recommendations $(80 \%)$, and clients may abuse the use of chatbots and self-assess too often ( $80 \%)$. 


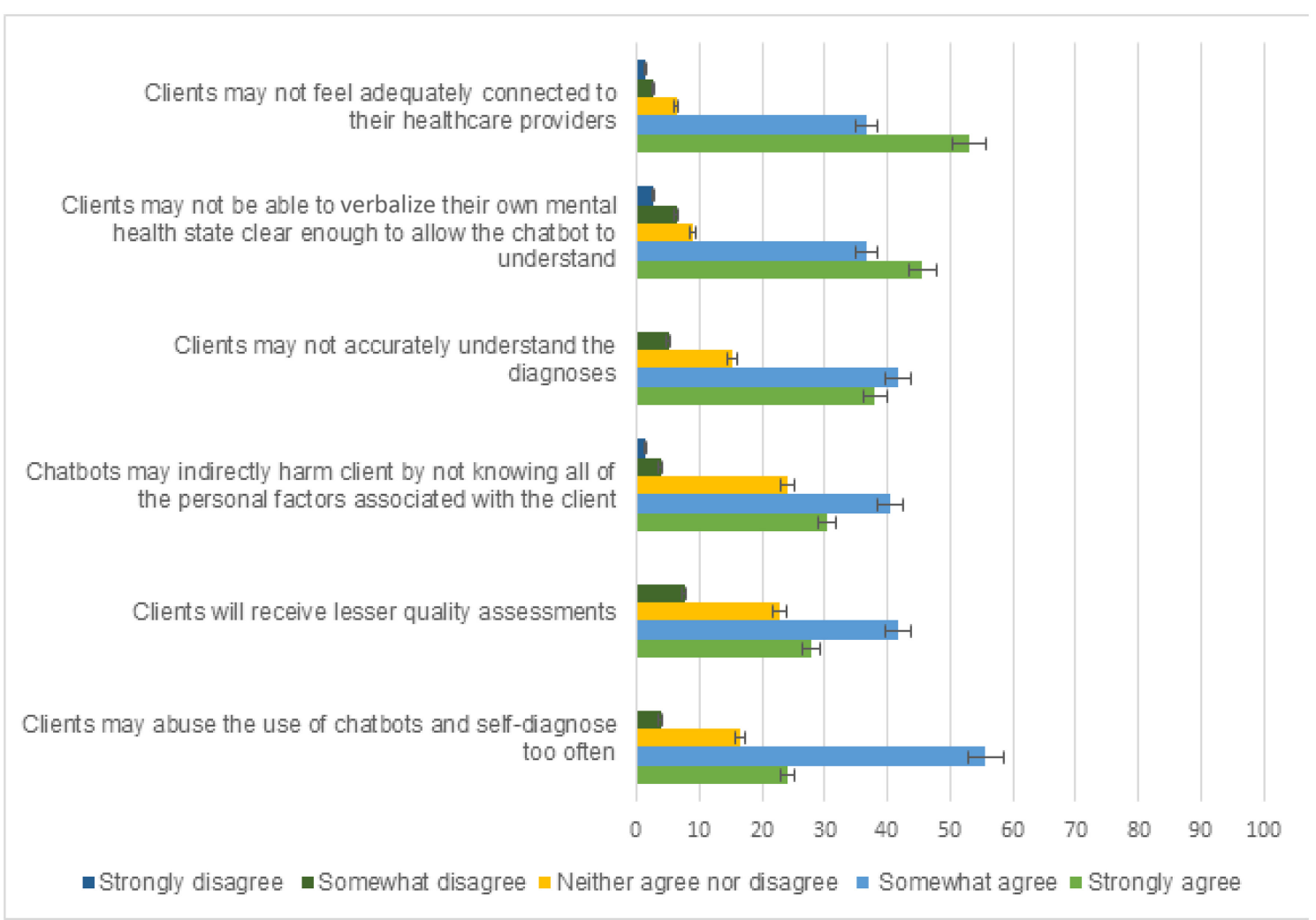

Fig. 6. Perceived risks associated with using mental healthcare chatbots for clients (ordered in descending order by percentage who strongly agree).

More than $57 \%$ of survey respondents indicated that healthcare chatbots would be more likely in the future to play a more significant role in the health of clients than the healthcare provider. This perhaps indicates that national health services should consider procuring mental health apps from specialized app development providers. Countries such as the United Kingdom and Sweden, where healthcare provision is free of charge at the point of care, are beginning to put in place mental health service apps libraries within their systems of universal health coverage.

From the survey results, it is evident that the majority of respondents agree that there are benefits associated with mental healthcare chatbots and that the perceived importance of chatbots is also relatively high.

Spearman's rank correlation coefficient was used to correlate the years of experience of the respondents, as mental healthcare professionals, to various perceptions they may have about the use of chatbots. As years of experience increased, there was a corresponding increase in the belief that mental healthcare chatbots could benefit people in specific areas of mental health management to help clients better manage their own mental health $(r=0.543, p<0.01)$, improve quality of mental healthcare of people $(r=0.536, p<0.01)$, improve access and timeliness to mental healthcare $(\mathrm{r}=0.557, \mathrm{p}<0.01)$, and assist mental healthcare workers in their daily occupational role $(\mathrm{r}=0.547, \mathrm{p}<0.01)$. There were also significant statistical correlations between increased experience and a likelihood to recommend the prescription of mental healthcare chatbots to healthcare provider colleagues $(r=0.224, p<0.03)$, and a likelihood to prescribe the use of mental healthcare chatbots to clients within the next 5 years $(\mathrm{r}=0.219, \mathrm{p}<0.03)$. All of these correlations are statistically significant $(\mathrm{p}<0.05)$, and 
the results indicate that as people's experience increases, so does their belief that the use of chatbots can improve the quality of care, client self-management, access to care, and assist mental healthcare workers in their roles. Similarly, there is an increase in likelihood to both recommend and prescribe the use of chatbots with increasing years of experience of the mental healthcare professional.

\section{DISCUSSION}

\subsection{Principal Findings}

The primary aim of this study is to assess the feasibility and efficacy of chatbot use in the area of mental health, to assess the awareness, attitudes, and practices of mental healthcare professionals regarding the use of chatbots in improving mental health service provision, while also determining if there is any association between a person's experience and their belief that the use of chatbots can improve the quality of care, client self-management, access to care, and assist mental healthcare workers in their roles. An additional objective is to determine if there is any association between a person's experience and mental health professionals' likelihood to recommend or prescribe the use of mental healthcare chatbots to clients. A total of 100 respondents from Northern Ireland, Ireland, Scotland, Sweden, and Finland took part in this online survey, and all respondents were either professionals or experts in the area of mental health.

In relation to physical, psychological, or behavioral health benefits to clients, the respondents believe that mental healthcare chatbots could increase activity or exercise, improve nutrition or diet, reduce stress, and improve medication or treatment adherence. Respondents also found chatbots would be beneficial as reminders for treatment compliance, renewing medication prescriptions, and answering medication Frequently Asked Questions. Perceived logistical benefits of using mental healthcare chatbots show a strong percentage believing that there are advantages for organization, planning, and management of administrative characteristics associated with mental healthcare.

The work by Palanica et al. [24] indicated that chatbots may play a beneficial role in healthcare. However, the present study has developed significantly on that research with the primary finding from this study indicating that with greater experience, mental healthcare professionals have greater belief in the use of chatbots to better manage their clients' own mental health. There was a significant statistical correlation between increased experience and a likelihood to recommend the prescription of mental healthcare chatbots to healthcare provider colleagues, and a likelihood to prescribe the use of mental healthcare chatbots to clients within the next 5 years. This could be seen as an unexpected result where the expectation would be that younger respondents (with less experience) would be more embracing of new technologies, but counter-intuitively, people with more experience (who are generally older) are shown to have belief that these tools have a place in the mental healthcare process, and they would be more likely than healthcare workers with less experience to recommend or prescribe the use of mental healthcare chatbots to their clients. Even though there are significant statistical correlations between both increased experience and age with a likelihood to prescribe the use of mental healthcare chatbots to clients within the next 5 years, this is not a clear picture across all age groups. Further analysis has shown that $50 \%$ of respondents younger than 30 years have responded that they would be very likely to prescribe the use of mental healthcare chatbots to their clients within the next 5 years, compared to $24 \%$ for 30 to 39 year olds, $42 \%$ for 40 to 49 year olds, $18 \%$ for 50 to 59 year olds, and $33 \%$ for those older than 60 . If "very likely" and "somewhat likely" responses are combined, then $75 \%$ of respondents younger than 30 years have responded that they would be very likely or somewhat likely to prescribe the use of mental health care chatbots to their clients within the next 5 years, compared to $80 \%$ for 30 to 39 year olds, $79 \%$ for 40 to 49 year olds, $77 \%$ for 50 to 59 year olds, and $83 \%$ for those older than 60 .

Similar to the findings of a previous cross-sectional web-based survey, which examined the physicians' perceptions of chatbot use in general healthcare, the mental health professional's adoption of available chatbots is less extensive than anticipated. Even so, more than half of the respondents to the survey agreed to some extent that 
there are benefits associated with mental healthcare chatbots, yet the perceived adoption among clients remains quite low. This reflects some of the results from a recent trial by Fitzpatrick et al. [10], where they found that despite the effectiveness of these applications, the adoption and adherence to these tools is relatively poor [10]. The perceived importance of chatbots was also relatively high with more than three-quarters of respondents agreeing that mental healthcare chatbots could help their clients better manage their own health. Chatbots were also perceived to improve access and timeliness to care, reduce travel time to their mental healthcare providers, and prevent unnecessary visits to mental healthcare providers. The mental healthcare workers also believed that clients may disclose more information to chatbots compared to mental healthcare providers.

As well as perceived benefits, challenges and risks also exist. The main challenge identified with the use of mental healthcare chatbots was in relation to chatbots not adequately understanding or displaying human emotion. When trying to replicate the duties of a (human) expert, it was perceived that chatbots lack the intelligence or knowledge to assess clients accurately, and it is believed that chatbots cannot effectively care to the full extent of the clients' needs. Another area that needs to be addressed in terms of chatbot adoption relates to data privacy, as the respondents perceived client data privacy and confidentiality as challenge. An overwhelming majority of the respondents $(90 \%)$ claimed that their clients may not feel adequately connected to their healthcare providers when using chatbots, and these challenges and concerns would need to be addressed before the technology can be fully endorsed by the mental healthcare workers and adopted.

\subsection{Implications for Policy and Practice}

There are many implications for the development and deployment of chatbot services in the area of mental healthcare. Development could focus more on the self-management, education, and training elements of the application to reflect the findings of this survey, to direct policy and practice. The developers of these applications should consider what the mental healthcare workers find most beneficial about these applications or what could have the greatest potential. The perception of the use of some methods seems to increase with increased years of experience, which is an important finding. As such, the integration of these tools could involve training people together with people with more years' experience, who are not as digitally adept but have a belief in the role of technology. Chatbots have been perceived to relieve certain barriers in mental health provision, which includes stigma around accessing psychological health services and geographical isolation. Findings from this study show that respondents agree that mental healthcare chatbots could help clients better manage their own health, improve access and timeliness to care, reduce travel time to mental healthcare providers, and prevent unnecessary visits to mental healthcare providers. Either more pilot projects would be needed to demonstrate the technological soundness of some of these applications or a national review board would be needed to review and rate mental healthcare apps. One solution to this is the Organisation for the Review of Care and Health Applications (ORCHA), which is providing health app evaluation, where a greater than $65 \%$ rating is a precondition for adoption by the UK's National Health Service (NHS), for example. ORCHA outlines that there are three main challenges to digital health solutions, which include lack of awareness of the part that these applications can play in the management of health and care related conditions, difficulty in accessing appropriate apps for specific needs, and the lack of trust that is inhibiting the utilization of digital health solutions.

There are also some uncertainties and concerns about the impact of chatbots in mental healthcare provision. Although there was a general perception that chatbots could potentially be used to help clients better manage their own health, more than $25 \%$ (27.5\%) did not believe that clients would be inclined to disclose more information to chatbots compared to mental healthcare providers. Similarly, the respondents saw problems in the areas of diagnosing (51\%), counseling (32.5\%), and cognitive behavioral therapy (25\%), possibly because these could be perceived as replacements for vital roles within mental healthcare management. Only when these tools can be developed to meet the requirements of the people who will use them, and are practiced in real-life settings, will their impact be fully realized. Developers should take these findings into consideration when developing 
mental healthcare chatbots, such as to focus on integrating reminders into the software, for example, relating to the client's goals, medication, and exercise and use the chatbots to help alleviate stress and educate people. As the mental healthcare workers also believed that clients would disclose more information to chatbots compared to mental healthcare providers, the developer needs to be able to assure client users about privacy and confidentiality. The development of these mental healthcare chatbots could also focus on increasing activity or exercise or work toward reducing stress or improving medication or treatment adherence. Finally, chatbots may offer an opportunity to redesign the mental healthcare system in such a way that promotes genuine collaboration between mental healthcare professionals and technologists. To integrate these new technologies into the area of mental health provision successfully, mental healthcare professionals and policymakers need to appraise the reliability and validity of such tools. If scientific evidence shows the benefits of these tools, policymakers will then need to investigate the ethical and legal concerns associated with the use of artificial intelligence to support mental health counseling treatment programs so that these new methods can be successfully implemented.

\subsection{Limitations}

One of the limitations of this research is that the sample number is relatively small $(\mathrm{n}=100)$. All of the chatbots mentioned as examples in the survey were in English language and thus not necessarily familiar to professionals in Finland and Sweden, which is a limitation. The survey also did not capture the age group of the clients with whom the mental health professionals worked, which is a limitation and an area for future research. A further limitation is the sampling method used. The snowball survey sampling method, also known as convenience sampling, will not guarantee that a random sample is selected, as the people are recruited based on whom they know and their links to the ChatPal project, and therefore are not representative of the entire population. In addition to the majority of respondents being linked to a single project, there could also be a bias toward specific countries that may have a different attitude toward digital technology for mental healthcare compared with other countries.

\section{CONCLUSION}

This study shows that where chatbots have been used, the experience has been mostly satisfactory, even though the level of personal experience with chatbots among professionals and experts in mental health has been quite low. The results of this survey show that the majority of respondents agree that there are benefits associated with mental healthcare chatbots, and the perceived importance of chatbots is also relatively high. The perception is that clients may disclose more information to chatbots compared to mental healthcare providers, and in relation to physical/psychological/behavioral health benefits to clients, the respondents believe that mental healthcare chatbots could increase activity or exercise, improve nutrition or diet, reduce stress, and improve medication or treatment adherence.

Preliminary evidence for use of chatbots in the area of mental healthcare provision is favorable. This study has found that as years of experience increased, there was a corresponding increase in the belief that healthcare chatbots could help clients better manage their own mental health, improve quality of mental healthcare of people, improve access and timeliness to mental healthcare, and assist mental healthcare workers in their daily occupational role.

\section{ACKNOWLEDGEMENTS}

The authors would like to thank the clients, participants, project members, supporters, and researchers at Ulster University, University of Eastern Finland, Norrbotten Association of Local Authorities, Region Norrbotten, Luleå University of Technology, NHS Western Isles, Action Mental Health, Cork Institute of Technology, and Health Innovation Hub Ireland for the successful development, implementation, and evaluation of this research. The ChatPal project is supported by Interreg Northern Periphery and Arctic Programme under grant number 345 . 


\section{REFERENCES}

[1] Gerhard Andersson. 2016. Internet-delivered psychological treatments. Annual Review of Clinical Psychology 12 (2016), 157-179.

[2] R. R. Bond, Siobhan O’Neill, Edel Ennis, and Maurice Mulvenna. 2019. Chatbots for mental health and suicide prevention: Is this even ethical? In Proceedings of the European Conference on Mental Health.

[3] DanaKai Bradford and David Ireland. 2016. Virtually There: Chatbots for Mental Health support? Retrieved April 27, 2021 from https: //pdfs.semanticscholar.org/9dfe/eb34503a318d5c25fbd037258826e31dcd9f.pdf.

[4] Petter Bae Brandtzaeg and Asbjørn Følstad. 2017. Why people use chatbots. In Proceedings of the International Conference on Internet Science. 377-392.

[5] Gillian Cameron, David Cameron, Gavin Megaw, Raymond Bond, Maurice Mulvenna, Siobhan O'Neill, Cherie Armour, and Michael McTear. 2018. Assessing the usability of a chatbot for mental health care. In Proceedings of the International Conference on Internet Science. 121-132.

[6] G. Cameron, D. Cameron, G. Megaw, R. Bond, M. Mulvenna, S. O’Neill, C. Armour, and M. McTear. 2017. Towards a chatbot for digital counselling. In Proceedings of the 31st British Computer Society Human Computer Interaction Conference. Article 24, 7 pages.

[7] Helen Christensen, Kathleen M. Griffiths, and Louise Farrer. 2009. Adherence in Internet interventions for anxiety and depression: Systematic review. Journal of Medical Internet Research 11, 2 (2009), e13.

[8] Kyungyong Chung and Roy C. Park. 2019. Chatbot-based heathcare service with a knowledge base for cloud computing. Cluster Computing 22, 1 (2019), 1925-1937.

[9] Kevin Cullen. 2018. eMental Health State-of-the-Art \& Opportunities for Ireland. Technical Report. Work Research Centre. https://www. mentalhealthreform.ie/wp-content/uploads/2018/10/eMental-Health-State-of-the-art-Opportunities-for-Ireland-Full-Report.pdf.

[10] Kathleen Kara Fitzpatrick, Alison Darcy, and Molly Vierhile. 2017. Delivering cognitive behavior therapy to young adults with symptoms of depression and anxiety using a fully automated conversational agent (Woebot): A randomized controlled trial. FMIR Mental Health 4, 2 (2017), e19.

[11] Asbjørn Følstad and Petter Bae Brandtzæg. 2017. Chatbots and the new world of HCI. Interactions 24, 4 (2017), 38-42.

[12] Russell Fulmer, Angela Joerin, Breanna Gentile, Lysanne Lakerink, and Michiel Rauws. 2018. Using psychological artificial intelligence (Tess) to relieve symptoms of depression and anxiety: Randomized controlled trial. FMIR Mental Health 5, 4 (2018), e64.

[13] Adam C. Jaroszewski, Robert R. Morris, and Matthew K. Nock. 2019. Randomized controlled trial of an online machine learning-driven risk assessment and intervention platform for increasing the use of crisis services. Fournal of Consulting and Clinical Psychology 87, 4 (2019), 370.

[14] Tobias Kowatsch, Dirk Volland, Iris Shih, Dominik Rüegger, Florian Künzler, Filipe Barata, Andreas Filler, et al. 2017. Design and evaluation of a mobile chat app for the open source behavioral health intervention platform MobileCoach. In Proceedings of the International Conference on Design Science Research in Information System and Technology. 485-489.

[15] Kira Kretzschmar, Holly Tyroll, Gabriela Pavarini, Arianna Manzini, Ilina Singh, and NeurOx Young People's Advisory Group. 2019. Can your phone be your therapist? Young people's ethical perspectives on the use of fully automated conversational agents (chatbots) in mental health support. Biomedical Informatics Insights 11 (2019), 1178222619829083.

[16] Lisa A. Chalaguine, Fiona L. Hamilton, Anthony Hunter, and Henry W. W. Potts. 2018. Argument harvesting using chatbots. Computational Models of Argument: Proceedings of COMMA 2018305 (2018), 149.

[17] Gale M. Lucas, Albert Rizzo, Jonathan Gratch, Stefan Scherer, Giota Stratou, Jill Boberg, and Louis-Philippe Morency. 2017. Reporting mental health symptoms: Breaking down barriers to care with virtual human interviewers. Frontiers in Robotics and AI 4 (2017), 51.

[18] Claudia Martinez and Imogen Farhan. 2019. Making the right choices. Retrieved April 27, 2021 from https://www.bl.uk/britishlibrary/ /media/bl/global/social-welfare/pdfs/non-secure/m/a/k/making-right-choices-using-data-driven\%20technology-19.pdf.

[19] Patrick McAllister, James Kerr, Michael McTear, Maurice Mulvenna, Raymond Bond, Karen Kirby, Joseph Morning, and Danni Glover. 2020. Towards chatbots to support bibliotherapy preparation and delivery. In Chatbot Research and Design, Asbjørn Følstad, Theo Araujo, Symeon Papadopoulos, Effie Lai-Chong Law, Ole-Christoffer Granmo, Ewa Luger, and Petter Bae Brandtzaeg (Eds.). Springer International, Cham, Switzerland, 127-142.

[20] Adam Miner, Amanda Chow, Sarah Adler, Ilia Zaitsev, Paul Tero, Alison Darcy, and Andreas Paepcke. 2016. Conversational agents and mental health: Theory-informed assessment of language and affect. In Proceedings of the 4th International Conference on Human Agent Interaction. 123-130.

[21] Adam S. Miner, Arnold Milstein, and Jefferey T. Hancock. 2017. Talking to machines about personal mental health problems. FAMA 318, 13 (2017), 1217-1218.

[22] David C. Mohr, Michelle Nicole Burns, Stephen M. Schueller, Gregory Clarke, and Michael Klinkman. 2013. Behavioral intervention technologies: Evidence review and recommendations for future research in mental health. General Hospital Psychiatry 35, 4 (2013), 332-338.

[23] Megan Molteni. 2017. The chatbot therapist will see you now. Science-Wired Magazine. Retrieved April 27, 2021 from https://www. wired.com/2017/06/facebook-messenger-woebot-chatbot-therapist. 
[24] Adam Palanica, Peter Flaschner, Anirudh Thommandram, Michael Li, and Yan Fossat. 2019. Physicians' perceptions of chatbots in health care: Cross-sectional web-based survey. Journal of Medical Internet Research 21, 4 (2019), e12887.

[25] Sami Pirkola, Heidi Eriksen, Erkki Isometsä, Aino Joensuu, Pekka Jylhä, Virve Kekkonen, Hannu Koponen, et al. 2020. Suicide Prevention and Suicide Attempt. Current Care Recommendation. Technical Report. Finnish General Medical Association, Helsinki. https://www. kaypahoito.fi/en/ccs00130.

[26] Kristian Pollock, Sarah Armstrong, Catherine Coveney, and John Moore. 2010. An Evaluation of Samaritans Telephone and Email Emotional Support Service. University of Nottingham, Nottingham, UK.

[27] Nicole M. Radziwill and Morgan C. Benton. 2017. Evaluating quality of chatbots and intelligent conversational agents. arXiv:1704.04579.

[28] Marita Skjuve and Petter Bae Brandtzæg. 2018. Chatbots as a new user interface for providing health information to young people. In Youth and News in a Digital Media Environment: Nordic-Baltic Perspectives, Y. Andersson, U. Dalquist, and J. Ohlsson (Eds.). Nordicom, Goteborg, Sweden, 59-66.

[29] Aditya Nrusimha Vaidyam, Hannah Wisniewski, John David Halamka, Matcheri S. Kashavan, and John Blake Torous. 2019. Chatbots and conversational agents in mental health: A review of the psychiatric landscape. Canadian fournal of Psychiatry 64, 7 (2019), $456-464$.

[30] J. Weizenbaum. 1965. ElizaA computer program for the study of natural language communication between man and machine. Communications of the ACM 9, 36 (1965), 45.

[31] Julia Shuppert West, Lynda Kayser, Paul Overton, and Robert Saltmarsh. 1991. Student perceptions that inhibit the initiation of counseling. School Counselor 39, 2 (1991), 77-83.

[32] Rainer Winkler and Matthias Söllner. 2018. Unleashing the potential of chatbots in education: A state-of-the-art analysis. Retrieved April 27, 2021 from https://www.alexandria.unisg.ch/254848/1/JML_699.pdf.

Received April 2020; revised October 2020; accepted February 2021 